OPEN ACCESS

Edited by:

Felix Putze,

University of Bremen, Germany

Reviewed by:

Michael Tangermann

Albert Ludwigs University of Freiburg,

Germany

Ricardo Chavarriaga,

École Polytechnique Fédérale de

Lausanne, Switzerland

${ }^{*}$ Correspondence:

Irina-Emilia Nicolae

irina.nicolae@aut.pub.ro

Benjamin Blankertz

benjamin.blankertz@tu-berlin.de

Specialty section:

This article was submitted to

Neuroprosthetics,

a section of the journal

Frontiers in Neuroscience

Received: 20 July 2017 Accepted: 20 September 2017

Published: 04 October 2017

Citation:

Nicolae $1-E$, Acqualagna $L$ and Blankertz B (2017) Assessing the Depth of Cognitive Processing as the

Basis for Potential User-State

Adaptation. Front. Neurosci. 11:548.

doi: $10.3389 /$ fnins.2017.00548

\section{Assessing the Depth of Cognitive Processing as the Basis for Potential User-State Adaptation}

\author{
Irina-Emilia Nicolae ${ }^{1,2 *}$, Laura Acqualagna ${ }^{2}$ and Benjamin Blankertz ${ }^{2 \star}$ \\ ${ }^{1}$ Department of Applied Electronics and Information Engineering, Politehnica University of Bucharest, Bucharest, Romania, \\ ${ }^{2}$ Department of Neurotechnology, Technische Universität Berlin, Berlin, Germany
}

Objective: Decoding neurocognitive processes on a single-trial basis with Brain-Computer Interface ( $\mathrm{BCl}$ ) techniques can reveal the user's internal interpretation of the current situation. Such information can potentially be exploited to make devices and interfaces more user aware. In this line of research, we took a further step by studying neural correlates of different levels of cognitive processes and developing a method that allows to quantify how deeply presented information is processed in the brain.

Methods/Approach: Seventeen participants took part in an EEG study in which we evaluated different levels of cognitive processing (no processing, shallow, and deep processing) within three distinct domains (memory, language, and visual imagination). Our investigations showed gradual differences in the amplitudes of event-related potentials (ERPs) and in the extend and duration of event-related desynchronization (ERD) which both correlate with task difficulty. We performed multi-modal classification to map the measured correlates of neurocognitive processing to the corresponding level of processing.

Results: Successful classification of the neural components was achieved, which reflects the level of cognitive processing performed by the participants. The results show performances above chance level for each participant and a mean performance of 70-90\% for all conditions and classification pairs.

Significance: The successful estimation of the level of cognition on a single-trial basis supports the feasibility of user-state adaptation based on ongoing neural activity. There is a variety of potential use cases such as: a user-friendly adaptive design of an interface or the development of assistance systems in safety critical workplaces.

Keywords: encephalography (EEG), event-related potentials (ERPs), oscillatory activity, cognitive processes, single-trial classification

\section{INTRODUCTION}

While Brain-Computer Interface (BCI) research primarily targets medical applications (Birbaumer, 2006; Dornhege et al., 2007; van Gerven et al., 2009; Wolpaw and Wolpaw, 2012; Guger et al., 2014; Hassanien and Taher Azar, 2015), more and more perspectives are being explored that go beyond communication and control paradigms (Blankertz et al., 2010, 2015, 2016; Zander and Kothe, 2011; Borghini et al., 2014; Zander et al., 2016). One of those perspectives are systems that take the 
ongoing user mental state into account and automatically adapt according to the user's mindset (Müller et al., 2008; van Erp et al., 2015) exploiting implicit information (Gamberini et al., 2015).

Neural correlates of cognitive processes can be found in the time-locked Event-Related Potentials (ERPs), usually composed of different components, and in modulations of spontaneous brain rhythms. The phase of those background rhythms is not time-locked to external stimuli, but the modulation of their amplitudes (resp. their hull curves) can be time-locked. In that case, the effect is called Event-Related Desynchronization or Synchronization (ERD/ERS) (Pfurtscheller and Lopes da Silva, 1999; Lemm et al., 2009), depending on whether it is a decrease or an increase in spectral power of the given frequency band.

ERP components have been widely investigated and previous studies involving cognitive activities in oddball paradigms show that the amplitude and latency of the ERPs are modified according to task difficulty (Donchin et al., 1973; Ullsperger et al., 1987; Polich, 2007; Kim et al., 2008). Specifically, increased P300 amplitude and longer latencies are usually found, relating to more complex processes and stronger attentional demand. These cognitive ERP components are reported to appear between 300$500 \mathrm{~ms}$ after the stimuli, in the centro-parietal cortex (Polich, 2007).

Moreover, changes in frequency are also reported, being modulated by the difficulty of cognitive processes. Firstly, the presentation of a stimulus triggers a short synchronization proceeded by a prolonged desynchronization mostly occurring in the $\alpha$ band at the frontal, temporal, central, and parietal locations of the scalp in accordance with the type of cognitive process (Klimesch et al., 1992, 1993, 1997; Klimesch, 1999), and follows the P300 potential (Yordanova et al., 2001). The $\alpha$ band desynchronizes during mental activity and cognitive judgment (Klimesch, 1999), and is reported proportionally increasing with more difficult cognitive processing, change mostly encountered at the centro-parietal sites. The effect is visible in laboratory environments (Gevins et al., 1997) and as well in more realistic scenarios (Venthur et al., 2010).

In addition, $\beta$ oscillations are also linked to complex cognitive processes (Pesonen et al., 2007; Okazaki et al., 2008; Sheth et al., 2009), whereas decreased $\beta$ oscillations appearing at the central and parietal sites are correlated to complex reasoning (Basile et al., 2013), decision making (Nakata et al., 2013) and are also related to the transition of cognitive states (Sheth et al., 2009).

Changes in the $\theta$ activity are observed as synchronizations in relation to task difficulty (Klimesch, 1999), e.g., increased $\theta$ power proportionally with increased memory load (Gundel and Wilson, 1992; Gevins et al., 1997), which also relate to the encoding of new information (Klimesch et al., 1996; Klimesch, 1999). Regarding localization, the $\theta$ changes are known to appear at the frontal midline scalp location (Gevins et al., 1997).

A pronounced ERD was found in relation to different types of cognitive processes. For example, in memory processes (Mecklinger et al., 1992; Klimesch et al., 1994; Stipacek et al., 2003; Pesonen et al., 2007), perceptual encoding and attentional processes, a stronger ERD is observed in the $\alpha$ band (Sergeant et al., 1987; Klimesch, 2003; Schack et al., 2005; Polich, 2007).
In addition, the processing of semantic information, e.g., words, shows likewise ERD enhancements (Klimesch et al., 1997).

In a recent study, Naumann et al. (2017), investigated gradual differences in task difficulty by estimating the difficulty level of a video game from the ongoing neural activity of the user. They found likewise significant modulations in the $\theta(4-7 \mathrm{~Hz})$ and $\alpha(8-13 \mathrm{~Hz})$ frequency bands, associated with changes in task difficulty.

In the present work, we have studied the feasibility of quantifying how deeply presented information is processed in the brain by tapping the corresponding components of brain activity. To that end, we analyzed single-trial EEG data with respect to its discriminative value of ERPs and of modulations of brain rhythms. In the ERPs, we found as main effect an increase of the P300 amplitude with task demand and also domain specific modulations in later components, see also (Nicolae et al., 2015a). Spontaneous background oscillations showed a prolonged suppression of the $\alpha$ and $\beta$ rhythm as a reflection of profound cognitive processing. For extracting the features for classification, we employed spatio-temporal features of the ERPs (Blankertz et al., 2011) and we exploited the ERD effect by combining spatio-spectral decomposition (SSD, Nikulin et al., 2011) in two frequency bands with Common Spatial Patterns (CSP) analysis (Fukunaga, 1990; Koles, 1991). Combining features of ERPs and ERDs in a multimodal classification approach (Fazli et al., 2015) leads to a performance increase compared to using a single modality only (Nicolae et al., 2015b). The current manuscript comprises a detailed investigation of the depth of cognitive processing integrating the analysis over the modulations of brain rhythms (ERDs) and the spectral analysis discriminability (power spectrum, CSPs), extending previous work in Nicolae et al. (2015a), where the temporal and spatial evolution of the ERPs are briefly analyzed and the abstract in Nicolae et al. (2016), where a short overview over the power spectrum is presented. In addition, the present work contributes further with an enhanced multivariate classification approach based on both, the temporal and spectral features extracted from the EEG, as compared to the classification based on only the temporal features presented in (Nicolae et al., 2015b).

\section{MATERIALS AND METHODS}

\section{Experimental Setup}

\section{Participants}

Fifteen healthy participants with no acute or chronic neurological and/or psychiatric disorders and no pregnant women were considered for the study. Eleven participants were right-handed, ten were males and all were aged between 22 and 35 years old. Eleven participants had German as mother tongue, one participant had English and the others had different languages as native tongue, with a required good command of English or German in order to fulfill the task in the language condition. Seventeen participants were initially recorded, but two participant's data were removed for the analysis due to high artifacts probably caused by improper recordings. The experimental procedure was conducted in accordance with the declaration of Helsinki, approved by the ethics committee of the 
Department of Psychology and Ergonomics of the Technische Universität Berlin and written informed consent was obtained from each participant. To countenance participant's motivation, they received financial compensation.

\section{Material}

The hardware equipment used for the acquisition of the Electroencephalography (EEG) was a BrainAmp amplifier with 64 active electrodes (Brain Products $\mathrm{GmbH}$, Munich, Germany) positioned according to the 10-20 international system. One electrode, named EOG, was placed under the left eye and used for eye movements recording. We used unipolar recording at a sampling frequency of $1 \mathrm{kHz}$ with the ground placed on the scalp at position $\mathrm{AFz}$ and with reference at left mastoid. The acquisition system was re-referenced to left and right mastoids. When mounting the electrode cap, the impedance was kept below $20 \mathrm{k} \Omega$. The acquired data will be made available from the DepositOnce repository of Technische Universität Berlin (https://depositonce.tu-berlin.de/).

The stimuli were designed as vectorial graphics with the Inkscape software (version 0.91.0.1 https://inkscape.org) besides the images from the animals category which were created by Freepik and taken from the Freepik database (http://www.freepik. com/free-photos-vectors/icon). The stimuli were presented on a 24 " display with $60 \mathrm{~Hz}$ refresh rate and $1,920 \times 1,200$ resolution (Dell U2410). For developing the experimental paradigm, the Processing software (version 3.0a4 https://processing.org/) was used in conjunction with MATLAB software for signal acquisition (release R2014a, The MathWorks, Inc., Natick, MA, USA). For the offline analysis, the data was processed with the BBCI MATLAB Toolbox (https://github.com/bbci/bbci_public).

\section{Experimental Scenario}

We considered two degrees of cognitive processing, namely shallow and deep processing levels (Craik and Lockhart, 1972) in a visual stimuli paradigm. In our scenario, the shallow processing involves a basic information processing revealed by attention (color appearance) and deep processing requires a complex activity related to specific cognitive tasks in the memory, language, and visual imagination conditions (Ganis et al., 2013). Each visual stimulus consisted of a pair of two images, cartoonlike drawings and every image represented an object chosen out of three categories: animals, fruits and mobility and was represented in one of the colors: red, green, blue, or magenta. Both images had same color and same category, where each category consisted of a total of 10 objects, see Figure 1. In order to maintain the desired ratio and without increasing too much the difficulty of the tasks, only two out of the three categories were chosen for each run.

The experimental study took place in a laboratory environment. Participants performed the experiments seated, and were requested to stay still, relaxed, and focused. They were allowed to freely explore the information presented, but in general, to focus their view in the center of the screen. Each participant was preliminary prepared with a practice test (1-3 runs) in order to become familiar with the tasks.

The experiment paradigm is described below. Before the start of the sequence, and after a short personal current state evaluation, the condition to be performed was displayed along with the target image pair (target color and category). When the sequence started, participants had to distinguish first between color, and subsequently for category. If the color does not match the target, then no processing at all was requested (NonTarget, NT case). If only the color matched, perform only mental computation (Shallow Target, ST case). If color and category matched the target (Deep Target, DT case), then evaluate the requested cognitive task and perform the corresponding mental computations (addition). Following the example sketched in Figure 2, the procedure was as follows:

(1) Target pair (cue): remember the target category, target color and the target images;

(2) For each stimulus:

- First, check if the stimulus color matches the target color:

$\rightarrow$ if not, do nothing (NT-non-target);

$\rightarrow$ if yes, count +1 and:

- check if the category matches the target category:

$\rightarrow$ if not, do nothing (ST-shallow target);

$\rightarrow$ if yes, perform the cognitive task associated to each cognitive process and memorize the new images for the next trial in case of the memory condition:

- if the answer is negative, do nothing additional (DT-deep target);

- or in case of positive answer, additionally count $+10 \quad$ (DT-deep target);

The resulting final number was inserted at the end of each run, followed by the feedback regarding the correct number.

The cognitive conditions were separately conducted in this order: memory, language, and visual imagination, with five runs per condition. We did not alternate between the conditions after each run in order to avoid confusions between the tasks, which have been quite demanding. In each condition participants had to "answer" a yes-no question, which is detailed hereunder.

\section{Memory}

The first condition considers memory retrieval by comparing a previously presented stimulus with the current stimulus (Kirchner, 1958; Chen et al., 2008). Specifically, the question is whether the current stimulus coincides with previous target pair (last pair of target color and target category). The accomplishment implies memorization of the current pair and retrieval of the previous target part. For an example see Figure 2, left column. An additional example of the memory experimental paradigm is provided in Supplementary Video 1 of the Supplementary Presentation 1.

\section{Language}

The language task considered comparisons based on phonemic representations between the words that represent the images. The task was to decide whether the number of syllables of 

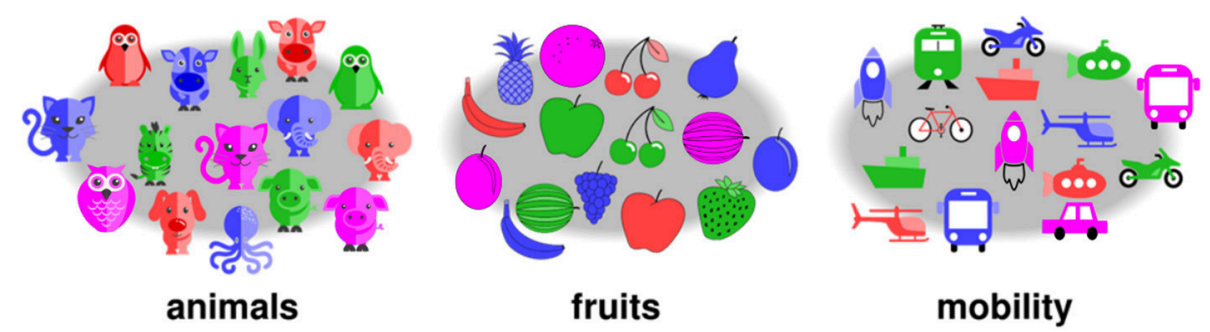

FIGURE 1 | Stimuli categories (animals, fruits, mobility) and objects representations examples. Each object could be presented in one of the four colors (red/green/blue/magenta).

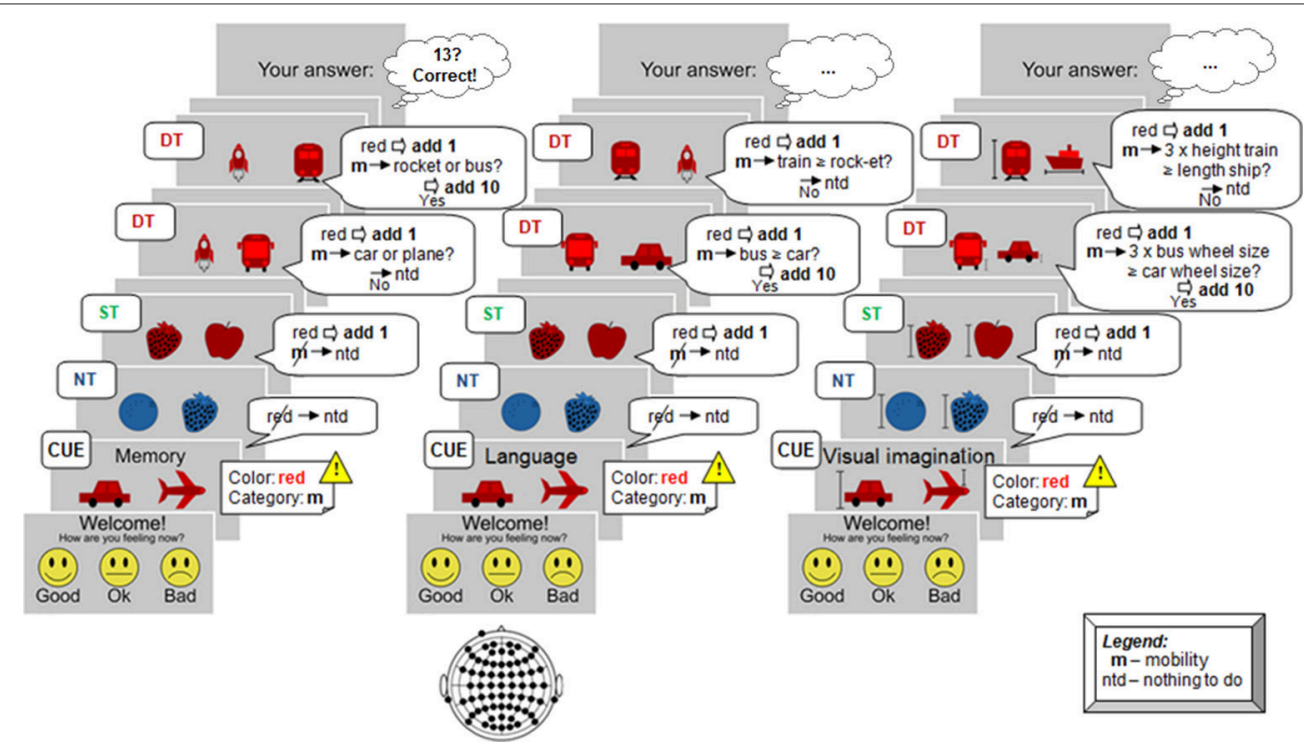

FIGURE 2 | Example of experimental protocol. At each stimulus participants had to perform or not a specific task, involving mental computation as a result. The total number counted was inserted at the end of the run. From left to right, the cognitive processes are illustrated here: memory case (recall memory and compare with the last target pair), language (compare the number of syllables), and visual imagination (imagine the objects in reality and perform dimensions comparison).

the left image's word was greater or equal than the number of syllables of the word for the object on the right side. This condition considered English or German words based on the participant's native language. Examples are shown in Figure 2, middle column. Note that the chosen objects (Figure 1) have a quite unique mapping to their representing words. In case a test person employed a different word, that could just affect the behavioral data, while the actual performance (DT vs. ST or NT) is not affected. Additional example for the language experimental paradigm is encountered in Supplementary Video 2 of the Supplementary Presentation 1.

\section{Visual imagination}

The visual imagination task required mental representations in order to perform a comparison based on the size in reality. The differentiations made are accomplished by judging whether three times the dimension of the left object (or a part of the object) is greater or equal to the right object's size, considering average dimensions of the represented objects. The respective part of the object and the dimension type (length, height, or thickness) used for comparison, was emphasized with a marker on the stimulus image. Further examples are in Figure 2, right column.

When generating the stimulus pairs for the visual imagination condition, a small constraint was added in order to ensure similar complexity within the visual imagination task. This related to the differences of dimensions within a range, more exactly the absolute difference between three times the left object and the right object should be less or equal than the left object dimension. Hence, no big discrepancy between the objects sizes was assured: no large differences implying an easy comparison, and no small ones either, which would result in a hard or ambiguous comparison. Short additional example for the visual imagination experimental paradigm is encountered in Supplementary Video 3 of the Supplementary Presentation 1.

\section{Experimental Design}

As a pilot study, four participants were asked to test the application without EEG cap. The results of this pilot study have been used to calibrate the speed and the complexity of the task. The time course of the experiment is presented in Figure 3. It 


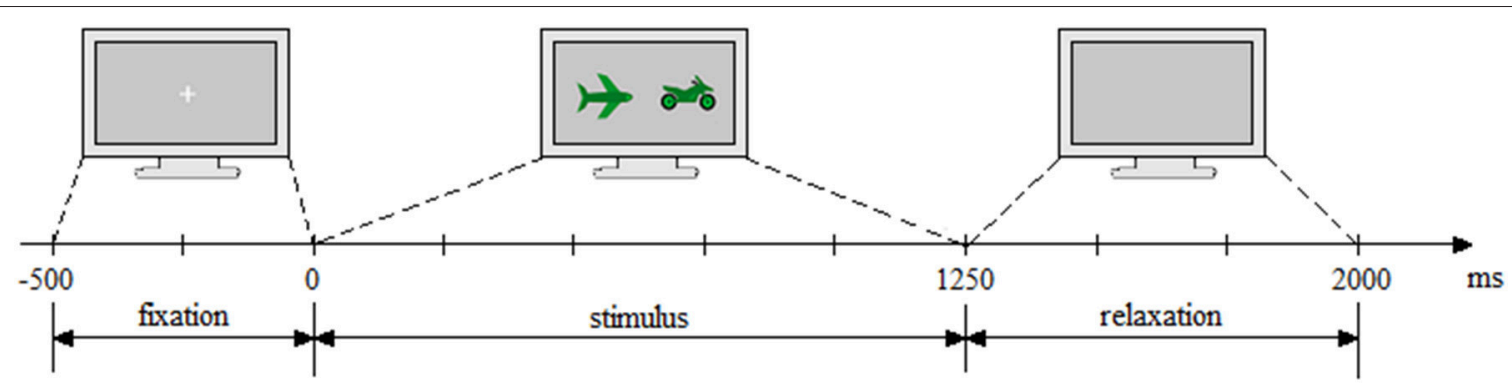

FIGURE 3 | Experiment timing. First, the fixation cross was displayed (500 ms), followed by the trial execution (1,250 ms) and ended with the relaxation period (750 ms, light gray blank screen).

consisted of 2,500 ms Inter-Stimuli Interval and was divided as follows: $500 \mathrm{~ms}$ fixation, 1,250 ms stimulus presentation, and 750 ms relaxation period.

Each condition was evaluated in five runs with a total of 600 stimuli. The two images representing the stimulus, were scaled to a common $480 \times 480$ resolution and presented close to the center of the screen at a distance of 2 " from one another on a light gray background.

The same percentage of shallow and deep targets was chosen in order to avoid confounds caused by a different number of occurrences: $75 \pm 2 \%$ for non-targets (NT) and $12.5 \pm 2 \%$ for both, shallow targets (ST) and deep targets (DT).

\section{Behavioral Assessment}

The subjects behavioral responses were assessed by subjective and objective indicators. The subjective indicators that we took into account by means of a questionnaire are: personal feedback, meaning personal internal state/mood (good/ok/bad) and personal overview for the difficulty of the conditions, which was acquired by scoring (0-easy; 1 -medium; 2 -hard). The objective indicator that we integrated (subjects answers ratio), is given by the actual responses, assessed by a ratio of the absolute difference between the correct number and user response number, divided by the correct number.

\section{Data Analysis}

Hereunder, the signal processing and machine learning methods are described, mainly the multi-modal analysis and multivariate classification which give us complementary and additional information over the neurophysiological effects of the cognitive processes. The multi-modal analysis investigates the neural correlates from the temporal and spectral domain: ERPs and Event-Related (De)Synchronization, ERDs. The brain sources of neural oscillations which substantiate cognitive activity (Varela et al., 2001; Buzsáki and Draguhn, 2004) were extracted by advanced decomposition methods: SSD by Nikulin et al. (2011) and Common Spatial Pattern (CSP) (Fukunaga, 1990). The two different types of neurophysiological information, temporal, and oscillatory activity, are combined with the concatenation approach as described in Dornhege et al. (2004), in order to give better performance as shown for example in other studies (Mühl et al., 2014). Finally, the depth of cognitive processing of the external information is estimated using multivariate singletrial classification, by Regularized Linear Discriminant Analysis (Friedman, 1989).

\section{Filtering and Epochs Rejection}

For dimensionality reduction, the data was downsampled to $100 \mathrm{~Hz}$. Preliminary processing of the data was performed by a sequence of low-pass filtering for anti-aliasing and high-pass filtering for drifts removal. The low-pass filter design for $42 \mathrm{~Hz}$, is a Chebyshev type II of order 10 with $42 \mathrm{~Hz}$ pass-band edge frequency and $3 \mathrm{~dB}$ ripple, and a $49 \mathrm{~Hz}$ stopband with $50 \mathrm{~dB}$ attenuation. For high-pass filtering, a $1 \mathrm{~Hz}$ FIR filter of order 300 (three times the downsampling frequency) was applied, using least-squares error minimization and reverse digital filtering with zero-phase effect (For future online classification, appropriate causal filters must be considered). Following, the data was segmented into epochs considering the experiment timing detailed in Figure 3.

A rough pre-cleaning of the data was additionally performed obviating noisy channels and epochs. A criterion evaluated on band-pass filtered data in a broad frequency band $(5-40 \mathrm{~Hz})$ was applied to remove the channels dropping to zero. Particularly, the channels with variance smaller than $0.5 \mu \mathrm{V}^{2}$ in more than $10 \%$ of the trials were removed. Moreover, the epochs with muscle artifacts were also removed considering the trials with excessive variance in $20 \%$ of the channels. For strong eye movements artifacts, max-min criterion was applied and epochs with more than $150 \mu \mathrm{V}$ difference between maximum and minimum voltage in channels F9, F10, AF3, and AF4 were removed. For removing the background noise of the remaining epochs, baseline correction was performed trial-wise by subtracting the mean amplitude computed on $100 \mathrm{~ms}$ of the pre-stimulus trial period from each trial period time point.

\section{Artifact Removal}

As a next step for removing the artifact data, including smaller eye movement artifacts, muscular artifacts and loose electrodes we performed Independent Component Analysis (ICA) with artifactual components selection given by the Multiple Artifact Rejection Algorithm (MARA, Winkler et al., 2011). For further verification, visual inspection was performed over each component considering the power spectral density and its topographic distribution. 


\section{Univariate Discriminative Analysis}

In addition to the temporal ERP analysis in Nicolae et al. (2015a), we evaluated the differences among levels of cognitive processing in the spectral domain (Nicolae et al., 2016). Moreover, we investigated the power spectrum with a discriminative measure given by the signed and squared point biserial correlation coefficient (signed $r^{2}$ ) which quantifies the discriminability between the two classes and the time course of power modulations with the help of Event-Related De/Synchronization (ERD/ERS) curves (Pfurtscheller and Lopes da Silva, 1999) in selected frequency bands.

\section{Multivariate Classification and Validation}

In order to join complementary information about the neural activity and therefore improve single-trial classification, we combined two different types of neurophysiological information. We considered spatio-temporal features reflecting ERPs and oscillatory activity features, which are described hereunder.

\section{Spatio-temporal features}

The spatio-temporal features (channels and time) were extracted as in Nicolae et al. (2015a). The method, further described in Blankertz et al. (2011), detects five temporal windows for each participant based on a heuristic selection of the intervals with maximum discriminability and a constant pattern between two classes based on the signed and squared point biserial correlation coefficient (signed $r^{2}$ ). The selected intervals contain the most significant spatio-temporal features, effective as found in other studies (e.g., Acqualagna and Blankertz, 2013).

\section{Spatio-spectral features}

As the cognitive processes produce observable modulations in the oscillatory activity, we considered extracting this information from the respective frequency bands. Based on the discriminative analysis, we selected the most significant frequency bands: $\alpha$ $(8-14 \mathrm{~Hz})$ and $\beta(16-20 \mathrm{~Hz})$. To enhance the discrimination of activity in the frequency band of interest, we performed SSD (Nikulin et al., 2011) and Common Spatial Pattern (CSP) (Blankertz et al., 2008) for the corresponding frequency bands.

Spatio-spectral decomposition, SSD. Linear spatial filtering prior to CSP was used in order to reach an efficient differentiation of mental states depicted by ERD/ERS rhythm patterns. Neural activity can be sometimes concealed in the background noise fluctuations, and therefore, for a better discrimination of the neural oscillations regarding cognitive processing, we applied SSD (Nikulin et al., 2011) which enhances the signal-to-noise ratio. In order to extract individual oscillatory sources, SSD finds the optimal spatial filters based on a generalized eigenvalue decomposition, that relates to high band power in the frequency of interest (pass-band filter) and low band power in the noise in the adjacent frequencies. The adjacent noise frequencies reduction is obtained with two pass band filters of a desired width (e.g., 1 or $2 \mathrm{~Hz}$ ) below and, respectively, above the frequency of interest, before or after a gap (stop-band filter of e.g., $1 \mathrm{~Hz}$ ) just below, respectively, just above the frequency of interest. Important notice: because SSD requires frequency filtering in advance, we used continuous data to avoid filter edges artifacts.
For the SSD decomposition, we consider only components with eigenvalues higher than $10^{-6}$ times the highest eigenvalue (see low-rank factorization in Haufe et al., 2014). Typically, between 15 and 35 components per discrimination pair were further selected.

Multi-band Common Spatial Patterns, mCSP. In order to obtain discriminative information about the cognitive processes based on oscillatory activity, we make use of the widely used CSP method as described by Fukunaga (1990) and Koles (1991), which was successively applied in a similar context (Schultze-Kraft et al., 2016). CSP facilitates the binary discrimination of different brain states by spatial filtering, enhancing the signal of interest while suppressing the background activity, by maximizing the variance for one class whilst minimizing the variance for the other class and vice versa. In our case, it increases the variance of a higher level of cognitive processing while diminishes the variance of a lower level of processing and vice versa. The components reaching this goal were automatically selected (as in Blankertz et al., 2008) up to a maximum of three spatial filters per class.

Based on the two relevant frequency bands, $\alpha(8-14 \mathrm{~Hz})$ and $\beta(16-20 \mathrm{~Hz})$, we extracted the most discriminative spatial filters within each band and we combined them, such that the neural components referring to both frequency bands could be simultaneously exploited. The entire process was performed on the data after applying band-pass and SSD filters. The time interval considered was selected from the ERD/ERS phenomena, starting from $350 \mathrm{~ms}$ after the stimuli, which corresponds roughly to the peak time point of the P300, from which point the cognitive process should generally begin (Nicolae et al., 2015a).

\section{Combined spatio-temporal and spatio-spectral features and evaluation scheme}

Targeting the estimation of user's cognitive processing, we followed the processing pipeline described in Figure 4. After appropriate preprocessing for each feature type (time or power), the spatio-temporal features were combined with the spatiospectral features given by the mCSP process, which were then classified and evaluated by cross-validation. More specifically, considering the band-power domain, the relevant spectral (logvariance) and spatial features were detected from the training data and used to spatially filter the testing data with the corresponding CSPs and were applied repeatedly in crossvalidation manner (10 folds with 10 repetitions). Further, the separation was performed by a regularized Linear Discriminant Analysis (Friedman, 1989; Lemm et al., 2011) with shrinkage of the covariance matrix (Ledoit and Wolf, 2004; Schäfer and Strimmer, 2005; Vidaurre et al., 2009; Blankertz et al., 2011), which proved to be successful for this type of analysis (Müller et al., 2003; Bartz and Müller, 2013; Farquhar and Hill, 2013). The classification performance, as the amount of correct estimated trials, was measured by the area under the Receiver Operating Characteristic (ROC) curve (Hanley and McNeil, 1982).

Initially, no normalization was performed on the feature vectors, because in our case, the features are roughly on the same scale. However, z-score normalization of the feature vectors was also applied as comparison, by subtracting the mean and dividing by the standard deviation on each feature type. 


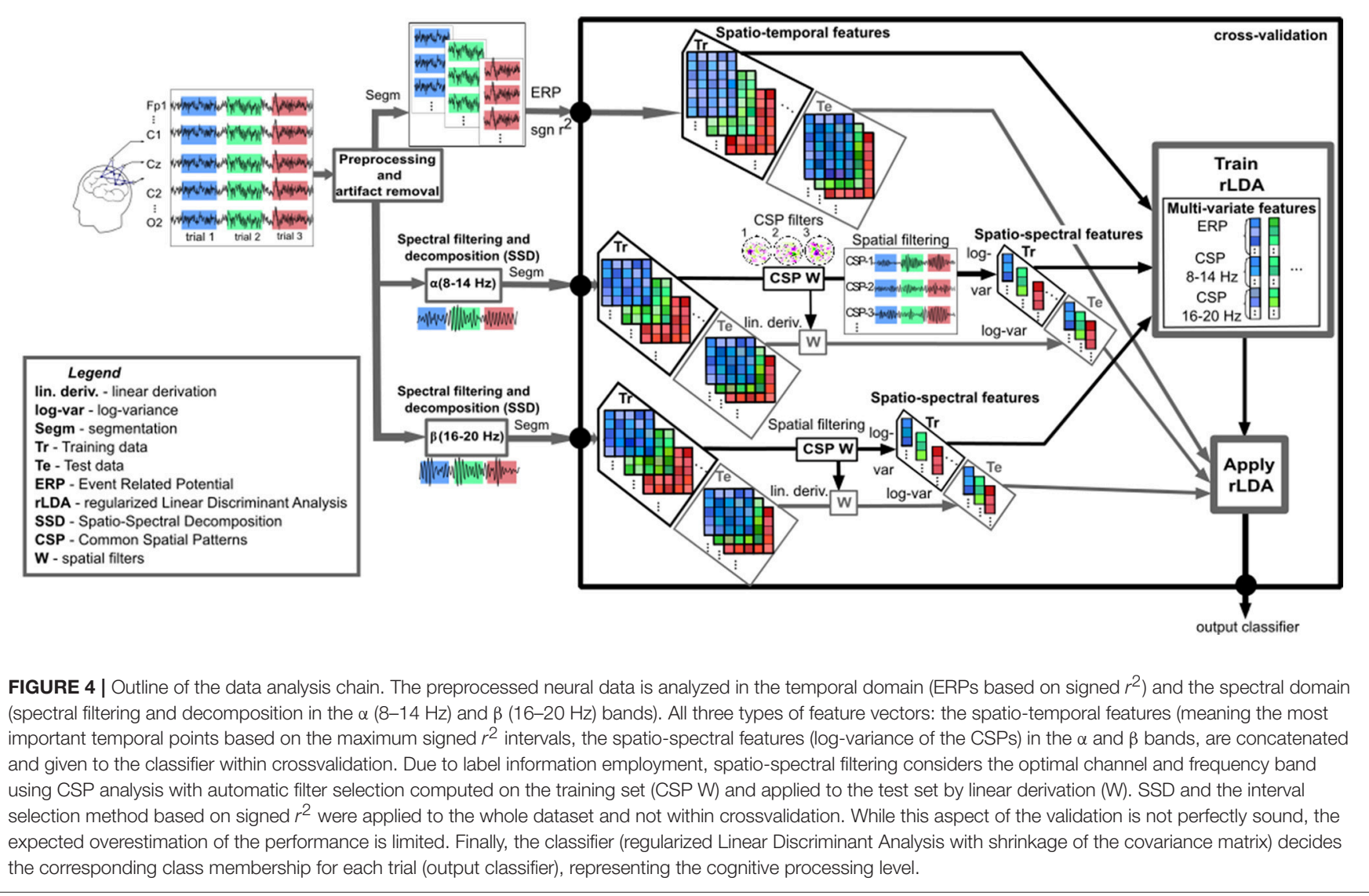

\section{RESULTS}

\section{Behavioral Data}

Regarding the subjective indicators about the difficulty of the conditions (Figure 5, right), the lowest score was attributed to the language condition, the $25 \%$ percentile shows 0 score difficulty and the $75 \%$ shows a medium difficulty with score 1 , meaning that the subjects mostly considered language as the easiest condition. The memory and visual imagination conditions were considered equally difficult by the subjects (25\% rated a medium difficulty with score 1 and around $75 \%$ rated a high difficulty with score 2 ). For the personal user mood evaluation, it was reported a good mood in 53\% of all the experiment runs and subjects, and in $47 \%$ it was specified as "ok." No bad mood was reported by the subjects during or after the experiment.

Considering the objective method given by answers ratio, we observe more accurate answers for the language condition with a ratio closer to zero (Figure 5, left-the answers ratio for all participants averaged over the runs for each condition). No improvement or decrease in performance was observed over time considering the answers ratio, showing insignificant correlations by the Spearman rank-order correlation (memory: $p=0.3367$; language $p=0.3982$; visual imagination $p=0.1211$; and in total over all 15 runs: $p=0.6192$ ). However, most of the participants showed engagement and enthusiasm throughout the experiment.

\section{Neurophysiological Data}

The temporal and spatial distribution of the neural activity represented by the ERPs is shown in Figure 6 (with more details in Nicolae et al., 2015a).

Looking at the spatial and temporal distribution of the ERPs in Figure 6, a gradual difference between the levels of cognitive processing is observed in the centro-parietal area reflecting a positive peak about $400 \mathrm{~ms}$ after the stimulus $(\sim \mathrm{P} 300)$. Earlier, a peak around $250 \mathrm{~ms}$ is observed, with a negative component more pronounced in the right-occipital cortex, discriminating between no processing and processing, and similarly in the visual imagination condition, but discriminating also between shallow and deep processing.

Second, we visualized the modulations of the signals' power spectrum computed for the entire trial timing (2s) in a spatialspectral representation (topographic maps). In order to focus on the discriminative aspects, the visualization was based on signed $r^{2}$-values.

For the distinction between shallow and deep processing (Figure 7, the bottom graphs), we investigated the average spectrum (mean over trials and participants) given by the signed $r^{2}$ over the parietal site $(\mathrm{Pz})$ in the frequency range from 3 to $40 \mathrm{~Hz}$. We observed a higher discriminative difference in the $\alpha$ band $(8-14 \mathrm{~Hz})$ and smaller in the $\beta$ band $(16-20 \mathrm{~Hz})$. Due to their prominent difference, also in the scalp maps at frontal and parietal sites, both frequency bands were selected for 

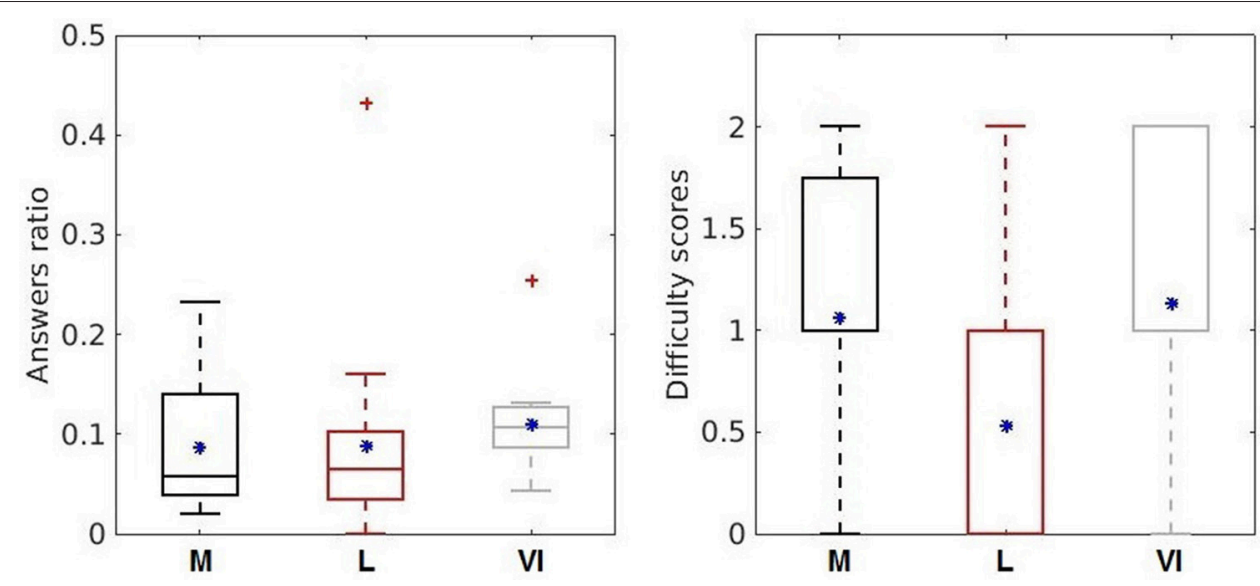

FIGURE 5 | Behavioral assessment indicators: answers ratio (left) and difficulty scores (right) for the three cognitive processes (memory - dark gray, language-light red, visual imagination-light gray). The upper and bottom whiskers of each box-plot corresponds to the maximum and minimum values over all participants. The horizontal sides of the rectangular boxes represent the 25 and $75 \%$ percentiles of the data. The mean values are represented by the blue asterisk ( ${ }^{*}$ ) and the outliers are indicated by the red crosses. Left plot of answers ratio is taken from Nicolae et al. (2015b) with permission from Springer.

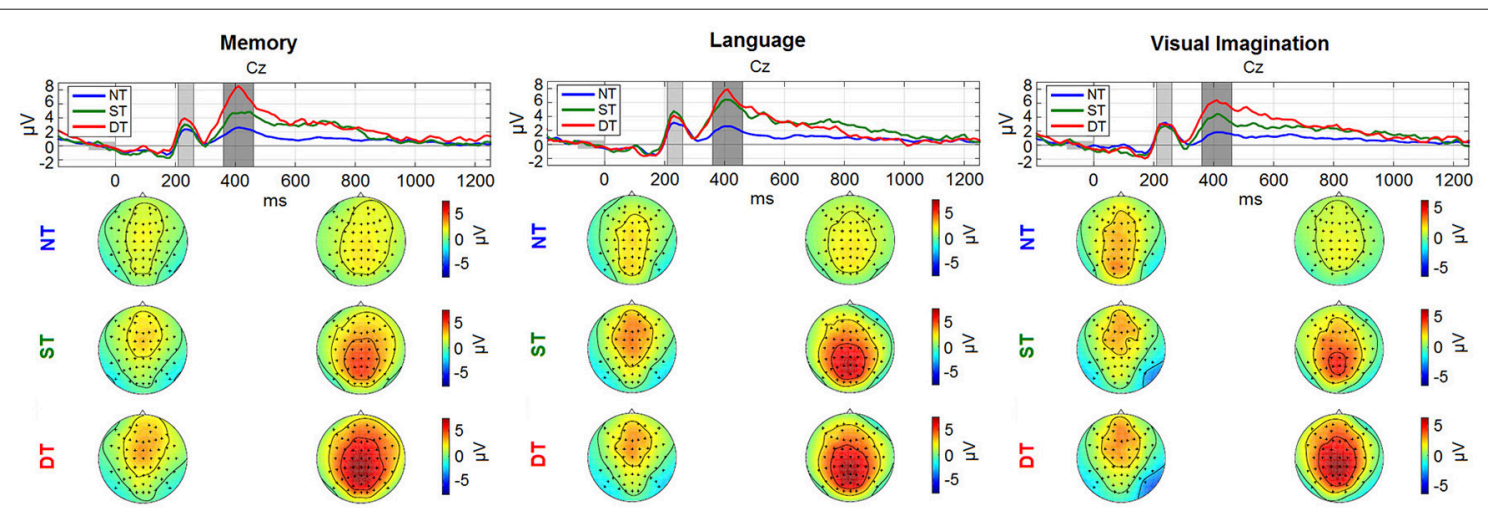

FIGURE 6 | Grand average ERPs: (a) Memory condition; (b) Language; (c) Visual imagination. The upper plots represent the time evolution at representative electrode $\mathrm{Cz}$ for the non-processing (blue line), shallow processing (green), deep processing (red). The scalp plots underneath show the topographies ( 2 to $8 \mu \mathrm{V}^{2}$ ) referring to the shaded areas (210-260 ms; 360-460 ms) highlighted in the time plots. The figure (with scaling adjustments) was taken from Nicolae et al. (2015a), with permission from the IEEE Proceedings.

the analysis in a multi-band approach. A modulation appears also in the $\theta$ band (5-7 Hz), visible when comparing shallow with no-processing and deep with shallow processing (upper and bottom graphs). This effect was less marked compared to the other frequency bands and it was not encountered in all levels of processing, therefore it was not considered for the analysis.

Figures 8, 9 depict the grand average desynchronization and synchronization effects in the $8-14 \mathrm{~Hz}$ and $16-20 \mathrm{~Hz}$ frequency band which start about $300 \mathrm{~ms}$ after stimulus. The time evolution is initially similar for all levels and conditions until $300 \mathrm{~ms}$, representing the same amount of evaluating the stimulus information. After this point, the effect of the desynchronization appears, climaxing around $500 \mathrm{~ms}$ and it follows a synchronization around $800-1,800 \mathrm{~ms}$. For visualization, we chose the central parietal electrode $\mathrm{Pz}$ (for other electrode patterns, see Supplementary Figures S1, S2 in Supplementary Presentation 1). It can be noticed that shallow or deep processes (DT) elicits an attenuation of brain rhythms in comparison to the reference of no-processing (NT). While the ERDs in the ST and DT levels start similarly, they are markedly more sustained in the DT level. The effect is more pronounced for the $\alpha$ band as -1 to $0.5 \mu \mathrm{V}$ and less for the $\beta$ band, as -0.4 to $0.4 \mu \mathrm{V}$. Looking over the scalp distributions, we clearly see higher synchronization (ERS) for the shallow processing $(0.1-0.35 \mu \mathrm{V})$ compared to the reference no-processing and more pronounced desynchronization (ERD) for a more complex processing $(-0.3-0.1 \mu \mathrm{V})$. Comparing between processes, a higher ERS in amplitude and spatial distribution is encountered for the memory process, contrasting to a more pronounced ERD in the language and visual imagination case.

Next, the CSP analysis provides a deeper view of the neural oscillations and activity related to cognitive processes. The patterns provide information about the presumed sources of the neural activity which are then optimally projected on the surface of the scalp. 

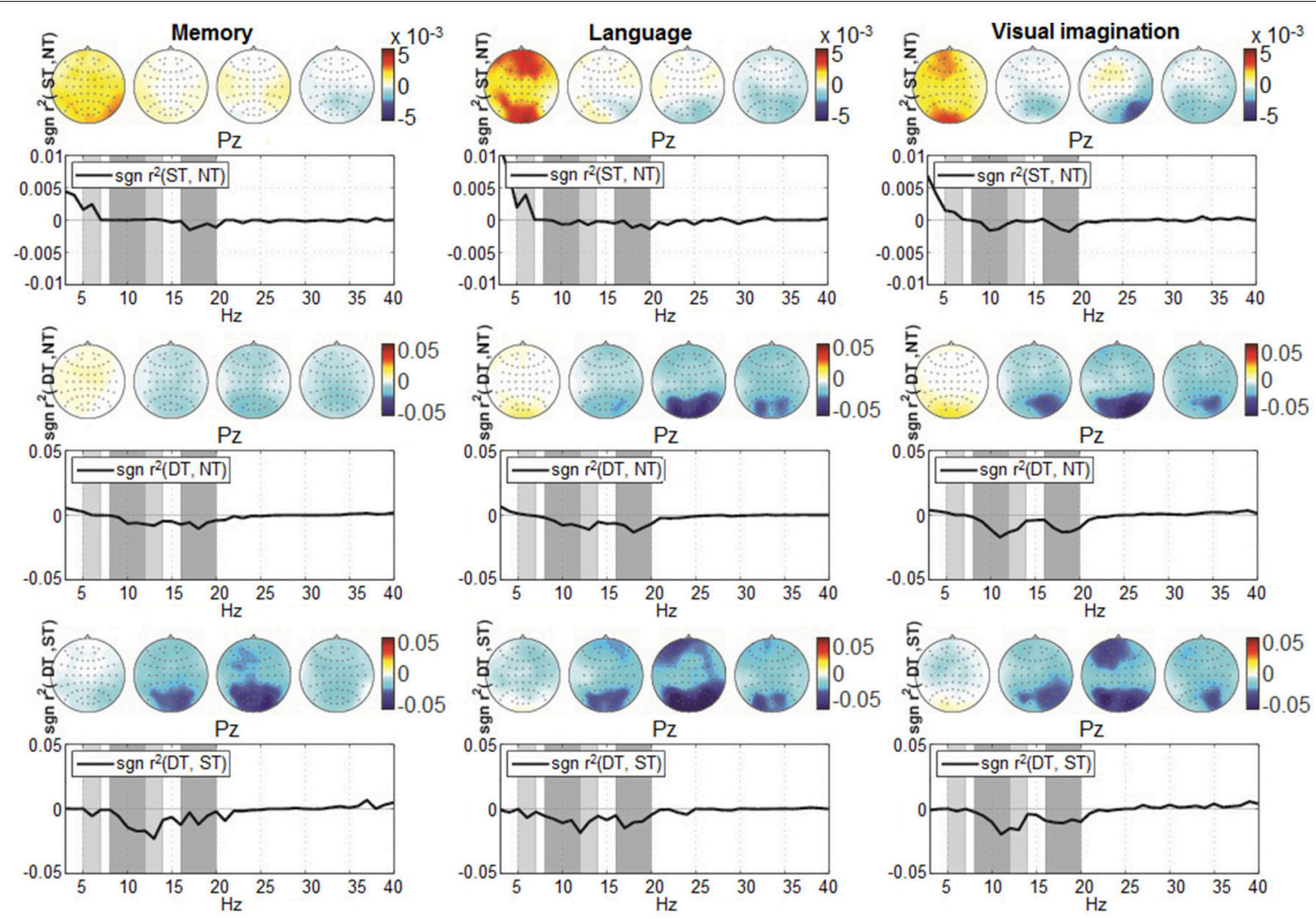

FIGURE 7 | Grand-average spectrum discrimination given by sgn $r^{2}$ at location Pz, computed over the entire trial timing (0-2,000 ms) in the frequency range from 3 to $40 \mathrm{~Hz}$. The discrimination pairs ST-NT, DT-NT, and DT-ST are represented from top to bottom, while from left to right are represented according to the three conditions: memory, language, and visual imagination. The four scalp plots refer to discriminative signed $r^{2}$-values, corresponding to $\theta$ (5-7 $\mathrm{Hz}$ ), $\alpha$ (8-12 $\mathrm{Hz}$; $12-14$ $\mathrm{Hz}$ ), $\beta(16-20 \mathrm{~Hz}$ ) frequency bands (shaded in gray). Note the upper graphs scale -0.01 to 0.01 , compared to the other scales -0.05 to 0.05 .
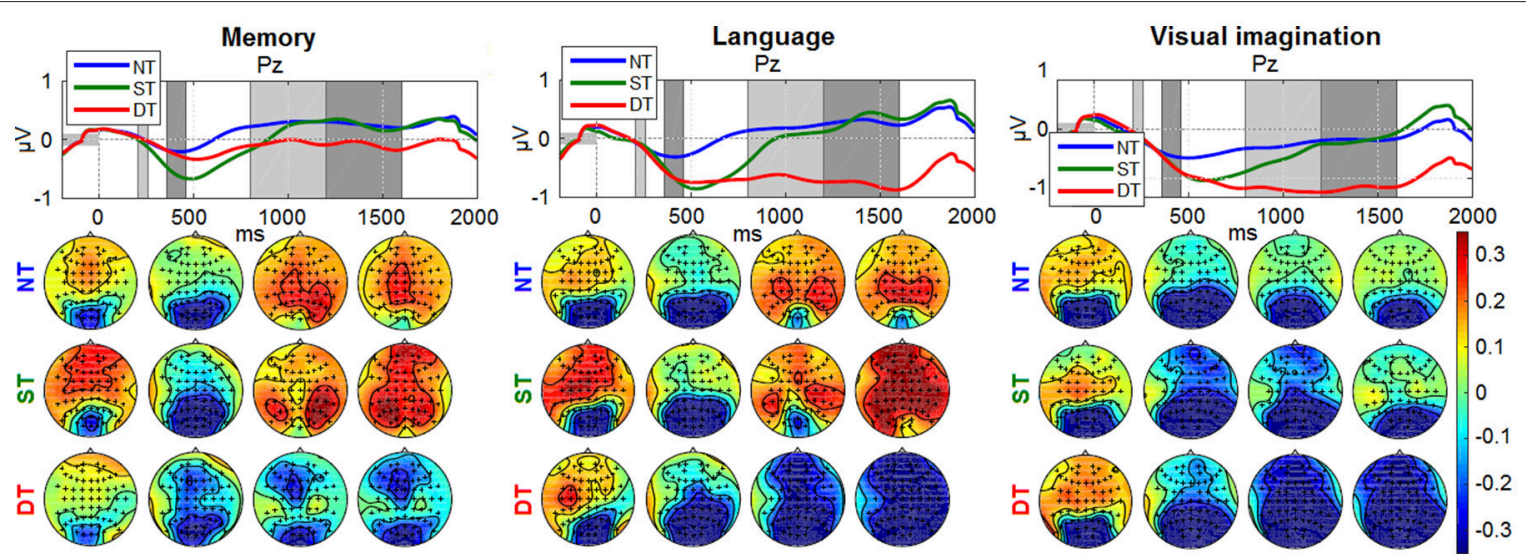

FIGURE 8 | Grand-average ERDs on 8-14 Hz for all processing levels (NT, ST, DT) at electrode Pz. The baseline interval is represented with a gray horizontal bar, equivalent to $200 \mathrm{~ms}$ of pre-stimulus interval. The amplitude range was chosen the same for all graphs, as -1 to $1 \mu \mathrm{V}$ for the time evolution and -0.35 to $0.35 \mu \mathrm{V}$ for the scalp distributions. The timing intervals for the scalp plots are: 210-260, 360-460, 800-1,200, 1,200-1,600 ms.

Considering the selected frequency bands, the CSP for participant P4 are shown in the Supplementary Presentation 1 as scalp topographies, computed for all pairs of class combinations (Supplementary Figures S3, S4).

\section{Classification}

The evaluation of the binary multivariate classification based on the combined spatio-temporal (ERP) and multi-band CSP (mCSP with SSD) features, are presented hereunder. The results 

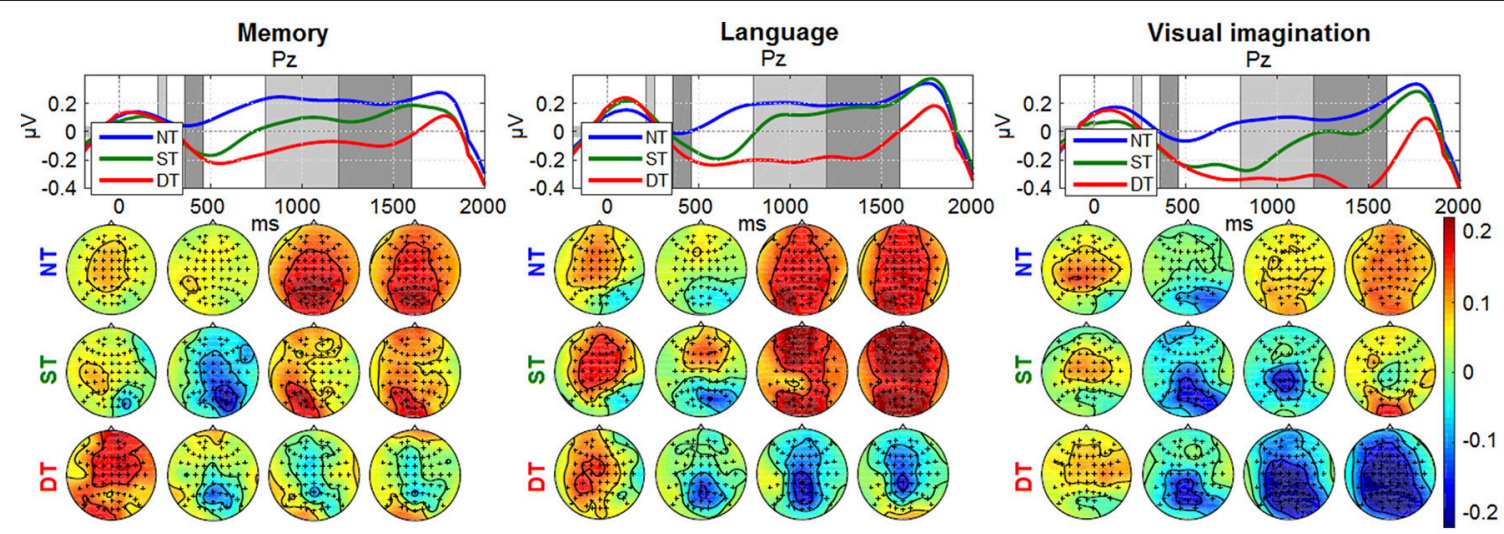

FIGURE 9 | Grand-average ERDs on 16-20 Hz at electrode Pz for all processing levels (NT, ST, DT) with $200 \mathrm{~ms}$ baseline. Same amplitude range for all conditions, as -0.4 to $0.4 \mu \mathrm{V}$ for time evolution and -0.22 to $0.22 \mu \mathrm{V}$ for scalp distributions.

of classification based on ERP only were presented in Nicolae et al. (2015b).

The general classification performance across participants given by the area under the ROC curve is presented as boxplots in Figure 10. Here we observe good performance which are on average above $70 \%$ for ST-DT discrimination, around $75-80 \%$ for NT-ST pair and the highest performance for NTDT discrimination, around $85-90 \%$. All performances for all participants are significantly above chance level (indicated by $t$ test with alpha $=0.01$ ). A two-way repeated measures ANOVA was performed over the AUC values with the factors: condition and classification pairs, which provide a statistically significant difference between the classification pairs $(p<0.001, F=$ 64.99). Based on the condition factor, the results in Figure 10 expose the highest average AUC for the language condition, but this observation was not statistically significant $(p=0.2112)$. The distribution of the data was verified using the one-sample Kolmogorov-Smirnov test, supposing the null hypothesis of standard normal distribution samples. The null hypothesis was rejected below the $1 \%$ significance level.

With standardization of the feature vectors, the performance is actually increased by $1-3 \%$, compared to the results in Figure 10 with no normalization, although this effect is not statistically significant ( $n$-way ANOVA: $p=0.0601$ ).

Now relating the classification results to the signed $r^{2}$ discrimination for the ERD/ERS curves, it is important to notice that even when no substantial difference was encountered for the NT-ST pair, the combined classification still performed considerably, which was due to the ensemble features approach that integrated also the temporal features. Moreover, when discriminating the deep processing, the classification is significantly improved in the presented combined approach compared to the separate temporal classification results (Nicolae et al., 2015b): increasing from 83 to $87 \%$ on average for the NTDT pair $(p=0.0027 ; F=9.57)$ and from 68 to about $72 \%$ for the ST-DT pair $(p=0.0234 ; F=5.33)$. No statistical significant difference was obtained for the NT-ST pair. Additionally, while considering only the spectral features, using the SSD method

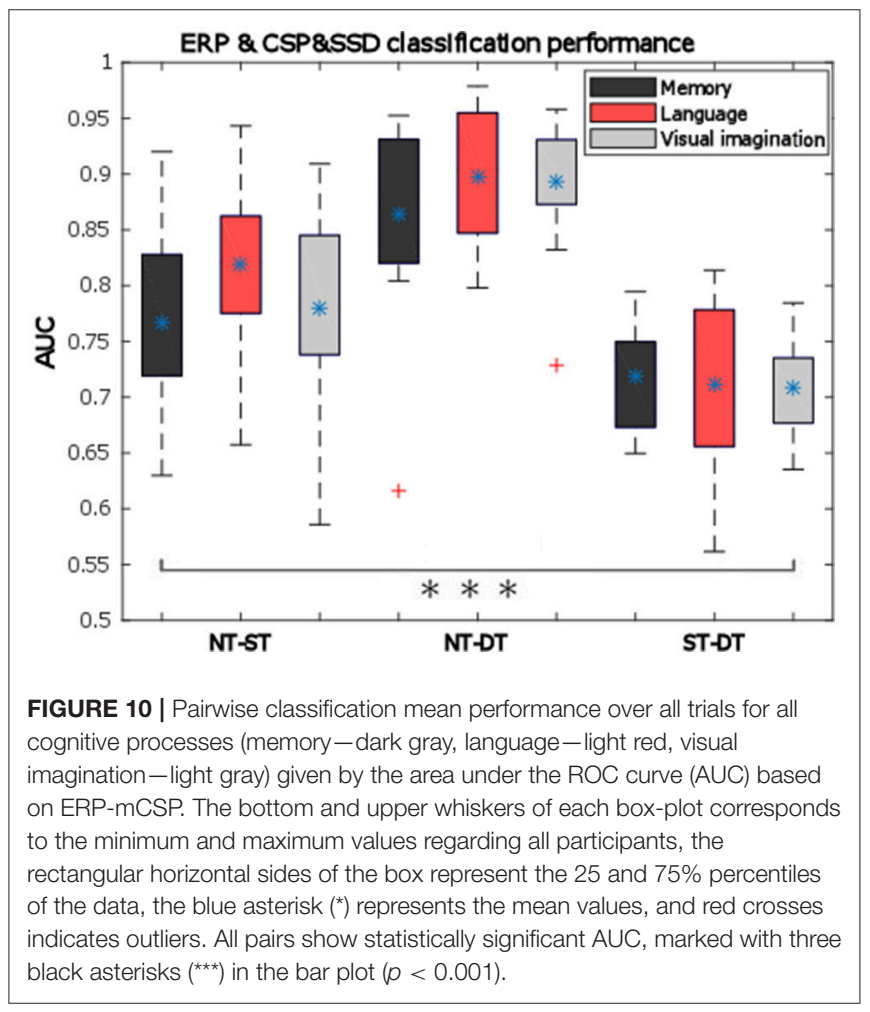

before CSP boosts the classification performance significantly for all cases: for example, from 61 to $74 \%$ on average for the STDT pair considering the visual imagination condition (statistically significant: $p=0.0001$ with the two-way ANOVA test).

\section{DISCUSSION AND CONCLUSION}

We investigated the neural correlates of cognitive processing that indicate the level of profoundness. Effects have been found in ERPs as well as in brain rhythms. 
In the ERPs, two peaks arise: one at $250 \mathrm{~ms}$ and one prolonged at $400 \mathrm{~ms}$. The first peak refers to the first decision on the type of the stimulus (NT, ST, or DT), based on appearance (color and category) which is similar between different levels of processing. the second peak relates to no processing (NT), mild (ST), or intense (DT) processing, and is graded in amplitude in relation to the corresponding level of processing. The latencies are similar because the levels of processing involve to the same decision task, mental computation. Further, in the deep processing task another decision is involved, deciding the task fulfillment or not, and hence different latencies will be observed trial to trial, which are not clearly visible on grand averages.

The power spectrum analysis, over a wide frequency range from 3 to $40 \mathrm{~Hz}$, provided an overview of the spectral components which were pronounced for the $\alpha(8-14 \mathrm{~Hz})$ and the $\beta$ band $(16-20 \mathrm{~Hz})$ and reduced for the $\theta$ band $(5-7 \mathrm{~Hz})$. Analyzing the components specific to each frequency band, we observed the present activity in the $\theta$ band being more pronounced for the memory and language condition, which reflects the memory retrieval activity (Meyer et al., 2015), cognitive processes (Klimesch, 1999), and sustained attention (Huang et al., 2007). However, the signed $r^{2}$ differences occurred in the $\theta$ band (see Figure 7) are substantially smaller than the differences occurred in the $\alpha$ and the $\beta$ band. Moreover, when testing the classification considering the $\theta$ band only, the performances were around chance level for all discrimination pairs, as expected (e.g., 0.51, 0.56, 0.51 mean AUCs for the language condition) which do not show significance with $t$-test at alpha $=0.0056$ with Bonferroni correction for multiple comparisons, given $p>0.0195$ for the NT-ST and ST-DT classification pairs and show barely significance for the NTDT discrimination with $p=0.0052$. Therefore, the decision was made to discard this band from further analysis, since in our case it did not produce significant differences. The ERD/ERS curves of the remaining frequency bands display a marked ERD with a peak at a latency of about $500 \mathrm{~ms}$. The duration of the ERD is modulated by the degree of cognitive processing. In addition, strong discrimination in the $\alpha$ band elucidates mental coordination (Palva and Palva, 2007), alert states (Klimesch, 1999), cognitive processing, access to stored information (Klimesch, 2012), and is completed with strong $\beta$ band differentiation that corresponds to complex mental process and analyzing the presented information (Lachaux et al., 2005).

Comparing across the conditions, the activity for memory is focused in the temporal and frontal area. More lateralized activity is observed for language and more accentuated in parietal and temporal area for visual imagination depicting memory access and interpretation (Ganis et al., 2004). Comparing the ERD/ERS effects between conditions, a more increased synchronization (higher DT in amplitude and power) is visible for the memory condition in comparison with the others, representing an easier process. This effect, contrasts with the behavioral point of view, where more participants stated, on average, the language as the easiest method (difficulty scores in Figure 5), effect found statistically significant with $p=0.0451$ given by a one-way ANOVA statistical test considering one factor (subjects) and three levels (conditions).
In order to improve the oscillatory discrimination, CSP filtering was employed to obtain spatial filters that distinguish the areas where the activity is differentiated between two processing levels (Supplementary Figures S3, S4 in the Supplementary Presentation 1).

For classification, we employed an approach which combines spatio-temporal features with spatio-spectral features. The average AUC is over $76 \%$ for the NT-ST pair, more than $86 \%$ for the NT-DT pair and $70 \%$ for the ST-DT pair. Note, that classification was challenged by the fact that participants moved their eyes freely between the two objects of each stimulus. In addition, it seems probably that the eye movements might have different dynamics between the tasks (e.g., DT might induce more alternations of the gaze between the two objects of a stimulus). For that reason, appropriate artifact removal strategies (ICA with MARA) and careful verification was performed. The resulting ERD and CSP patterns (cf. Figures 8, 9 and Supplementary Figures S3, S4 in Supplementary Presentation 1) do not suggest influences from eye movements, like strong lateralized activity in the frontal area which would be expected to result from horizontal movements. This gives a strong indication that the classification performance was not based on eye movement dynamics but on neural correlates of the cognitive processing. Furthermore, the evaluation of potential contamination by artifacts becomes more robust when analyzing the level of decoding based on the EOG activity alone. Specifically, the classification was performed considering two feature channels (EOG and Fp1; F10 and F9 channels difference) corresponding to the vertical and horizontal eye movements. The results show significant values at chance level for all conditions and classification pairs (e.g., mean AUCs for the language condition considering the classification pairs: $0.51,0.55,0.46)$ with $p>$ 0.0198 and Bonferroni corrected for the nine comparisons with alpha $=0.0056$.

In our experimental paradigm, different cognitive levels were externally imposed using task instructions. The graduated differences observed in the ERPs between shallow and deep processing correlate with different levels of processing, evidenced also in the ERDs/ERSs and are not generated by the targets occurrence, namely the odd-ball effect, which was controlled by imposing the same percentage of the stimuli between ST and DT (12.5\%). When comparing the shallow and deep processing with no processing, it cannot be disentangled which differences in the ERPs are generated by the rarity of the occurrence (oddball effect) and which by the additional processing demands of the task. In particular for the ERDs in Figure 8 the time course (differences extending far past $500 \mathrm{~ms}$ ) seems to suggest that the main difference is due to the additional cognitive processing in ST and DT. This design was chosen in order to have better control of the true level of cognitive processing. For the application perspective, however, we strive to estimate the momentary level of cognitive processing within the natural fluctuations.

In conclusion, the performed investigation of the depth of cognitive processing brings us closer to real scenarios. Compared to standard BCI research our study induced different levels of cognitive processing by tasks that go beyond a simple target/nontarget discrimination. Moreover, the visual stimuli used had 
a higher variability, including the need of eye movements in the exploration of the complex stimuli, which were composed of two objects side-by-side. Our work extends also previous investigation of the effect of task complexity on ERPs and brain oscillations. Again, the set of stimuli used in our study is richer and more complex and importantly, methods from machine learning have been employed for single-trial classification, in a binary approach.

Overall, the present study is a step forward toward applications that estimate the level of cognitive processing in realistic settings of human-computer interaction (Gamberini et al., 2015) and in safety critical workplaces (Venthur et al., 2010). A further step in order to discriminate the ongoing level of cognitive processing is to apply a regression approach, as in Naumann et al. (2017). A different perspective for the current study is the development of techniques suitable for adaptive learning environments based on user state monitoring regarding the depth of cognitive processing. In this regard, it would be interesting to augment the approach with predicting remembered vs. not remembered items (Klimesch et al., 1996) in memory tasks.

\section{DATA AVAILABILITY STATEMENT}

The EEG signals and behavioral data acquired in this study will be made available from the DepositOnce repository of Technische Universität Berlin (https://depositonce.tu-berlin.de/).

\section{AUTHOR CONTRIBUTIONS}

Design of the study by BB. Implementation and data acquisition by IN. IN performed data analysis and LA and BB reviewed and revised the analysis. IN wrote the manuscript draft which was reviewed and revised by $\mathrm{LA}$ and $\mathrm{BB}$.

\section{FUNDING}

Considering the research, we acknowledge financial support by the European Union Seventh Framework Programme (FP7/2007-2013) under grant agreement no. 611570, by the German Federal Ministry of Education and Research
(BMBF) under contract 01GQ0850 and by the Sectoral Operational Programme Human Resources Development 2007-2013 of the Ministry of European Funds through the Financial Agreement POSDRU/159/1.5/S/134398. Regarding publication, we acknowledge support by the German Research Foundation and the Open Access Publication Funds of Technische Universität Berlin.

\section{SUPPLEMENTARY MATERIAL}

The Supplementary Material for this article can be found online at: http://journal.frontiersin.org/article/10.3389/fnins. 2017.00548/full\#supplementary-material

Supplementary Video 1 | Short example with 30 trials for the experimental paradigm considering the memory condition. First, the visual questionnaire is presented evaluating participant's current mental state (good, ok, bad). Next, the target pair (pear, banana) specifying the target category (fruits) and the color target (red) is presented along with the current condition that needs to be performed (memory). When the participant memorized the target, he/she press a keyboard button and the sequence starts after a short countdown. At the end of the run, the participant enters the number counted and receives feedback considering the correct number. In total, there were 4 DT (from which two fulfilled the memory question task), 7 ST, and 19 NT stimuli presented, accumulating a final number counted of 31. Note that the number of trials is higher in the actual run of the experiment (120 stimuli) and the ratio of the stimuli is also different (75:12.5:12.5 \pm $2 \%$ for NT:ST:DT), while this example may seem harder (with a ratio of 63.3:23.3:13.3 (\%) for NT:ST:DT).

Supplementary Video 2 | Short example with 30 trials for the experimental paradigm considering the language condition. The target pair here (submarine, submarine) refer to the mobility category and shows the color target (green). The current condition that needs to be performed is also specified (language). In total, 4 DT (from which two fulfilled the language question task), 7 ST, and 19 NT stimuli were presented, resulting in a final number counted of 31. Same notice as in the memory example, that the number of trials and the ratio are different here compared with the actual experiment.

Supplementary Video 3 | Short example with 30 trials for the experimental paradigm considering the visual imagination condition. The target pair here (cow, elephant) and the color target (red) are presented along with the specification of the current condition that needs to be performed (visual imagination). In total, 4 DT (from which two fulfilled the visual imagination question task), 5 ST, and 21 NT stimuli were presented, resulting in a final number counted of 29. Same notice as in the memory and language example, that the number of trials and the ratio (70:16.67:13.3 (\%) for NT:ST:DT) are different compared with the actual experiment.

\section{REFERENCES}

Acqualagna, L., and Blankertz, B. (2013). Gaze-independent BCI-spelling using rapid serial visual presentation (RSVP). Clin. Neurophysiol. 124, 901-908. doi: 10.1016/j.clinph.2012.12.050

Bartz, D. M., and Müller, K. R. (2013). "Generalizing analytic shrinkage for arbitrary covariance structures," in Advances in Neural Information Processing Systems, Vol. 26 (Lake Tahoe), 1869-1877.

Basile, L. F., Sato, J. R., Alvarenga, M. Y., Henrique, N. Jr., Pasquini, H. A., Alfenas, W., et al. (2013). Lack of systematic topographic difference between attention and reasoning beta correlates. PLOS ONE 8:e59595. doi: 10.1371/journal.pone.0059595

Birbaumer, N. (2006). Breaking the silence: brain-computer interfaces (BCI) for communication and motor control. Psychophysiology 43, 517-532. doi: $10.1111 / \mathrm{j} .1469-8986.2006 .00456 . x$

Blankertz, B., Acqualagna, L., Dähne, S., Haufe, S., Schultze-Kraft, M., Sturm, I., et al. (2016). The Berlin brain-computer interface: progress beyond communication and control. Front. Neurosci. 10:530. doi: $10.3389 /$ fnins.2016.00530

Blankertz, B., Jacucci, G., Gamberini, L., Spagnolli, A., and Freeman, J. (eds.). (2015). "Symbiotic interaction," in 4th International Workshop, Symbiotic 2015, Proceedings (Cham: Springer International Publishing), 9359.

Blankertz, B., Lemm, S., Treder, M., Haufe, S., and Müller, K. R. (2011). Singletrial analysis and classification of ERP components-a tutorial. Neuroimage 56, 814-825. doi: 10.1016/j.neuroimage.2010.06.048

Blankertz, B., Tangermann, M., Vidaurre, C., Fazli, S., Sannelli, C., Haufe, S., et al. (2010). The Berlin brain-computer interface: non-medical uses of BCI technology. Front Neurosci. 4:198. doi: 10.3389/fnins.2010.00198

Blankertz, B., Tomioka, R., Lemm, S., Kawanabe, M., and Müller, K. R. (2008). Optimizing spatial filters for robust EEG single-trial analysis. IEEE Signal Process. Mag. 25, 41-56. doi: 10.1109/MSP.2008.4408441 
Borghini, G., Astolfi, L., Vecchiato, G., Mattia, D., and Babiloni, F. (2014). Measuring neurophysiological signals in aircraft pilots and car drivers for the assessment of mental workload, fatigue and drowsiness. Neurosci. Biobehav. Rev. 44, 58-75. doi: 10.1016/j.neubiorev.2012.10.003

Buzsáki, G., and Draguhn, A. (2004). Neuronal oscillations in cortical networks Science 304, 1926-1929. doi: 10.1126/science.1099745

Chen, Y. N., Mitra, S., and Schlaghecken, F. (2008). Sub-processes of working memory in the N-back task: an investigation using ERPs. Clin. Neurophysiol. 119, 1546-1559. doi: 10.1016/j.clinph.2008.03.003

Craik, F. I. M., and Lockhart, R. S. (1972). Levels of processing: a framework for memory research. J. Verbal Learn. Verbal Behav. 11, 671-684. doi: 10.1016/S0022-5371(72)80001-X

Donchin, E., Kubovy, M., Kutas, M., Johnson, R. Jr., and Herning, R. I. (1973). Graded changes in evoked response (P300) amplitude as a function of cognitive activity. Percept. Psychophys. 14, 319-324. doi: 10.3758/BF032 12398

Dornhege, G., Blankertz, B., Curio, G., and Müller, K. R. (2004). Boosting bit rates in non-invasive EEG single-trial classifications by feature combination and multi-class paradigms. IEEE Trans. Biomed. Eng. 51, 993-1002. doi: 10.1109/TBME.2004.827088

Dornhege, G., Millán, J. d. R., Hinterberger, T., McFarland, D., and Müller, K. R., (eds.). (2007). Toward Brain-Computer Interfacing. Cambridge MA: MIT Press.

Farquhar, J., and Hill, N. J. (2013). Interactions between pre-processing and classification methods for event-related-potential classification - best-practice guidelines for brain-computer interfacing. Neuroinformatics 11, 175-192. doi: 10.1007/s12021-012-9171-0

Fazli, S., Dähne, S., Samek, W., Bießmann, F., and Müller, K. R. (2015). Learning from more than one data source: data fusion techniques for sensorimotor rhythm-based brain-computer interfaces. Proc. IEEE. 103, 891-906. doi: 10.1109/JPROC.2015.2413993

Friedman, J. H. (1989). Regularized discriminant analysis. J. Am. Stat. Assoc. 84, 165-175. doi: 10.1080/01621459.1989.10478752

Fukunaga, K. (1990). Introduction to Statistical Pattern Recognition, 2nd Edn. Boston, MA: Academic Press.

Gamberini, L., Spagnolli, A., Blankertz, B., Kaski, S., Freeman, J., Acqualagna, L., et al. (2015). "Developing a symbiotic system for scientific information seeking: the mindsee project," in Symbiotic Interaction, Lecture Notes in Computer Science, Vol. 9359, eds B. Blankertz, G. Jacucci, L. Gamberini, A. Spagnolli, and J. Freeman (Cham, Switzerland: Springer International Publishing), 68-80.

Ganis, G., Lacey, S., and Lawson, R. (eds.). (2013). "Multisensory imagery," in Visual Mental Imagery, eds S. Lacey and R. Lawson (New York, NY: Springer Science Business Media LLC), 9-28.

Ganis, G., Thompson, W. L., and Kosslyna, S. M. (2004). Brain areas underlying visual mental imagery and visual perception: an fMRI study. Cogn. Brain Res. 20, 226-241. doi: 10.1016/j.cogbrainres.2004.02.012

Gevins, A., Smith, M. E., McEvoy, L., and Yu, D. (1997). High-resolution EEG mapping of cortical activation related to working memory: effects of task difficulty, type of processing, and practice. Cereb. Cortex 7, 374-385. doi: $10.1093 /$ cercor/7.4.374

Guger, C., Sorger, B., Noirhomme, Q., Naci, L., Monti, M. M., Real, R., et al. (2014). "Brain-computer interfaces for assessment and communication in disorders of consciousness," in Emerging Theory and Practice in Neuroprosthetics, ed G. R. Naik (Hershey, PA: IGI Global), 181-214.

Gundel, A., and Wilson, G. F. (1992). Topographical changes in the ongoing EEG related to the difficulty of mental tasks. Brain Topogr. 5, 17-25. doi: $10.1007 / \mathrm{BF} 01129966$

Hanley, J. A., and McNeil, B. J. (1982). The meaning and use of the area under a receiver operating characteristic (roc) curve. Radiology 143, 29-36. doi: 10.1148/radiology.143.1.7063747

Hassanien, A. E., and Taher Azar, A. (eds.) (2015). Brain-Computer Interfaces: Current Trends and Applications, Vol. 74. Springer.

Haufe, S., Dähne, S., and Nikulin, V. V. (2014). Dimensionality reduction for the analysis of brain oscillations. Neuroimage 101, 583-597. doi: 10.1016/j.neuroimage.2014.06.073

Huang, R. S., Jung, T. P., and Makeig, S. (2007). "Multi-scale EEG brain dynamics during sustained attention tasks," in Proceedings of the 32th International Conference on Acoustics, Speech and Signal Processing (Honolulu, HI), 1173-1176.
Kim, K. H., Kim, J. H., Yoona, J., and Jung, K. Y. (2008). Influence of task difficulty on the features of event-related potential during visual oddball task. Neurosci. Lett. 445, 179-183. doi: 10.1016/j.neulet.2008.09.004

Kirchner, W. K. (1958). Age differences in short-term retention of rapidly changing information. J. Exp. Psychol. 55, 352-358. doi: 10.1037/h0043688

Klimesch, W. (1999). EEG alpha and theta oscillations reflect cognitive and memory performance: a review and analysis. Brain Res. Rev. 29, 169-195. doi: 10.1016/S0165-0173(98)00056-3

Klimesch, W. (2003). "Interindividual differences in oscillatory EEG activity and cognitive performance," in The Cognitive Neuroscience of Individual Differences, eds I. Reinvang, M. W. Greenlee, and M. Herrmann (Oldenburg: BIS), 87-99.

Klimesch, W. (2012). Alpha-band oscillations, attention, and controlled access to stored information. Trends Cogn Sci. 16, 606-617. doi: 10.1016/j.tics.2012.10.007

Klimesch, W., Doppelmayr, M., Pachinger, T., and Russegger, H. (1997). Eventrelated desynchronization in the alpha band and the processing of semantic information. Cogn. Brain Res. 6, 83-94. doi: 10.1016/S0926-6410(97)00018-9

Klimesch, W., Doppelmayr, M., Russegger, H., and Pachinger, T. (1996). Theta band power in the human scalp EEG and the encoding of new information. Neuroreport 7, 1235-1240. doi: 10.1097/00001756-199605170-00002

Klimesch, W., Pfurtscheller, G., and Schimke, H. (1992). Pre- and poststimulus processes in category judgement tasks as measured by event-related desynchronization (ERD). J. Psychophysiol. 6, 185-203.

Klimesch, W., Schimke, H., and Pfurtscheller, G. (1993). Alpha frequency, cognitive load and memory performance. Brain Topogr. 5, 241-251. doi: 10.1007/BF01128991

Klimesch, W., Schimke, H., and Schwaiger, J. (1994). Episodic and semantic memory: an analysis in the EEG theta and alpha band. Electroenceph. Clin. Neurophysiol. 91, 428-441. doi: 10.1016/0013-4694(94)90164-3

Koles, Z. J. (1991). The quantitative extraction and topographic mapping of the abnormal components in the clinical EEG Electroencephalogr. Clin. Neurophysiol. 79, 440-447. doi: 10.1016/0013-4694(91)90163-X

Lachaux, J. P., George, N., Tallon-Baudry, C., Martinerie, J., Hugueville, L., Minotti, L., et al. (2005). The many faces of the gamma band response to complex visual stimuli. Neuroimage 25, 491-501. doi: 10.1016/j.neuroimage.2004.11.052

Ledoit, O., and Wolf, M. (2004). A well-conditioned estimator for large dimensional covariance matrices. J. Multivar. Anal. 88, 365-411. doi: 10.1016/S0047-259X(03)00096-4

Lemm, S., Blankertz, B., Dickhaus, T., and Müller, K. R. (2011). Introduction to machine learning for brain imaging. Neuroimage 56, 387-399. doi: 10.1016/j.neuroimage.2010.11.004

Lemm, S., Müller, K. R., and Curio, G. (2009). A generalized framework for quantifying the dynamics of Eeg event-related desynchronization. PLoS Comput. Biol. 5:e1000453. doi: 10.1371/journal.pcbi.1000453

Mecklinger, A., Kramer, A. F., and Strayer, D. L. (1992). Event-related potentials and EEG components in a semantic memory search task. Psychophysiology 29, 104-119. doi: 10.1111/j.1469-8986.1992.tb02021.x

Meyer, L., Grigutsch, M., Schmuck, N., Gaston, P., and Friederici, A. D. (2015). Frontal-posterior theta oscillations reflect memory retrieval during sentence comprehension. Cortex 71, 205-218. doi: 10.1016/j.cortex.2015.06.027

Mühl, C., Jeunet, C., and Lotte, F. (2014). EEG-based workload estimation across affective contexts. Front. Neurosci. 8:114. doi: 10.3389/fnins.2014.00114

Müller, K. R., Anderson, C. W., and Birch, G. E. (2003). Linear and nonlinear methods for brain-computer interfaces. IEEE Trans. Neural Syst. Rehabil. Eng. 11, 165-169. doi: 10.1109/TNSRE.2003.814484

Müller, K. R., Tangermann, M., Dornhege, G., Krauledat, M., Curio, G., and Blankertz, B. (2008). Machine learning for real-time single-trial EEG-analysis: from brain-computer interfacing to mental state monitoring. J. Neurosci. Methods 167, 82-90. doi: 10.1016/j.jneumeth.2007.09.022

Nakata, H., Sakamoto, K., Otsuka, A., Yumoto, M., and Kakigi, R. (2013). Cortical rhythm of No-go processing in humans: an MEG study. Clin. Neurophysiol. 124, 273-282. doi: 10.1016/j.clinph.2012.06.019

Naumann, L., Schultze-Kraft, M., Dähne, S., and Blankertz, B. (2017). "Prediction of difficulty levels in video games from ongoing EEG," in Symbiotic Interaction, Vol. 9961, eds L. Gamberini, A. Spagnolli, G. Jacucci, B. Blankertz, and J. Freeman (Cham: Springer International Publishing), 125-136.

Nicolae, I. E., Acqualagna, L., and Blankertz, B. (2015a). "Neural indicators of the depth of cognitive processing for user-adaptive neurotechnological 
applications," in Proceedings of International Conference of the IEEE Engineering in Medicine and Biology Society (Milano), 1484-1487.

Nicolae, I. E., Acqualagna, L., and Blankertz, B. (2015b). "Tapping neural correlates of the depth of cognitive processing for improving human computer interaction," in Symbiotic Interaction, Vol. 9359, Lecture Notes in Computer Science, eds B. Blankertz, G. Jacucci, L. Gamberini, A. Spagnolli, and J. Freeman (Cham: Springer International Publishing), 126-131. doi: 10.1007/978-3-319-24917-9_13

Nicolae, I. E., Acqualagna, L., and Blankertz, B. (2016). "Investigating depth of cognitive processing in the brain dynamics of oscillations," in Proceedings of 6th International BCI Meeting (Pacific Grove, CA: Verlag der TU Graz), 186.

Nikulin, V. V., Nolte, G., and Curio, G. (2011). A novel method for reliable and fast extraction of neuronal EEG/MEG oscillations on the basis of spatio-spectral decomposition. Neuroimage 55, 1528-1535. doi: 10.1016/j.neuroimage.2011.01.057

Okazaki, M., Kaneko, Y., Yumoto, M., and Arima, K. (2008). Perceptual change in response to a bistable picture increases neuromagnetic beta-band activities. Neurosci. Res. 61, 319-328. doi: 10.1016/j.neures.2008.03.010

Palva, S., and Palva, M. (2007). New vistas for $\alpha$-frequency band oscillations. Trends Neurosci. 30, 150-158. doi: 10.1016/j.tins.2007. 02.001

Pesonen, M., Hämäläinen, H., and Krause, C. M. (2007). Brain oscillatory 4-30 $\mathrm{Hz}$ responses during a visual $\mathrm{n}$-back memory task with varying memory load. Brain Res. 1138, 171-177. doi: 10.1016/j.brainres.2006.12.076

Pfurtscheller, G., and Lopes da Silva, F. H. (1999). Event-related EEG/MEG synchronization and desynchronization: basic principles. Clin. Neurophysiol. 110, 1842-1857. doi: 10.1016/S1388-2457(99)00141-8

Polich, J. (2007). Updating P300: an integrative theory of P3a and P3b. Clin. Neurophysiol. 118, 2128-2148. doi: 10.1016/j.clinph.2007.04.019

Schack, B., Klimesch, W., and Sauseng, P. (2005). Phase synchronization between theta and upper alpha oscillations in a working memory task. Int. J. Psychophysiol. 57, 105-114. doi: 10.1016/j.ijpsycho.2005.0 3.016

Schäfer, J., and Strimmer, K. (2005). A shrinkage approach to largescale covariance matrix estimation and implications for functional genomics. Stat. Appl. Genet. Mol. Biol. 4:32. doi: 10.2202/1544-6115.1175

Schultze-Kraft, M., Dähne, S., Gugler, M., Curio, G., and Blankertz, B. (2016). Unsupervised classification of operator workload from brain signals. J. Neural Eng. 13:036008. doi: 10.1088/1741-2560/13/3/036008

Sergeant, J., Geuze, R., and van Winsum, W. (1987). Event-related desynchronization and P300. Psychophysiology 24, 272-277. doi: 10.1111/j.1469-8986.1987.tb00294.x

Sheth, B. R., Sandkühler, S., and Bhattacharya, J. (2009). Posterior beta and anterior gamma oscillations predict cognitive insight. J. Cogn. Neurosci. 21, 1269-1279. doi: 10.1162/jocn.2009.21069

Stipacek, A., Grabner, R. H., Neuper, C., Fink, A., and Neubauer, A. C. (2003). Sensitivity of human EEG alpha band desynchronization to different working memory components and increasing levels of memory load. Neurosci. Lett. 353, 193-196. doi: 10.1016/j.neulet.2003.09.044

Ullsperger, P., Neumann, U., Gille, H. G., and Pietschmann, M. (1987). P300 and anticipated task difficulty. Int. J. Psychophysiol. 5, 145-149. doi: 10.1016/0167-8760(87)90018-3

van Erp, J. B., Brouwer, A. M., and Zander, T. O. (2015). Editorial: Using neurophysiological signals that reflect cognitive or affective state. Front. Neurosci. 9:36. doi: 10.3389/fnins.2015.00136

van Gerven, M., Farquhar, J., Schaefer, R., Vlek, R., Geuze, J., Nijholt, A, et al. (2009). The brain-computer interface cycle. J. Neural Eng. 6:041001. doi: 10.1088/1741-2560/6/4/041001

Varela, F., Lachaux, J. P., Rodriguez, E., and Martinerie, J. (2001). The brain web: phase synchronization and large-scale integration. Nat. Rev. Neurosci. 2, 229-239. doi: $10.1038 / 35067550$

Venthur, B., Blankertz, B., Gugler, M. F., and Curio, G. (2010). "Novel applications of BCI technology: psychophysiological optimization of working conditions in industry," in IEEE International Conference on Systems Man and Cybernetics (SMC) (Istanbul), 417-421.

Vidaurre, C., Krämer, N., Blankertz, B., and Schlögl, A. (2009). Time domain parameters as a feature for EEG-based brain-computer interfaces. Neural Netw. 22, 1313-1319. doi: 10.1016/j.neunet.2009.07.020

Winkler, I., Haufe, S., and Tangermann, M. (2011). Automatic classification of artifactual ICA-components for artifact removal in EEG signals. Behav. Brain Funct. 7:30. doi: 10.1186/1744-9081-7-30

Wolpaw, J. R., and Wolpaw, E. W. (eds.). (2012). Brain-Computer Interfaces: Principles and Practice. New York, NY: Oxford University Press.

Yordanova, J., Kolev, V., and Polich, J. (2001). P300 and alpha eventrelated desynchronization (ERD). Soc. Psychophysiol. Res. 38, 143-152. doi: $10.1111 / 1469-8986.3810143$

Zander, T. O., and Kothe, C. (2011). Towards passive brain-computer interfaces: applying brain-computer interface technology to human-machine systems in general. J. Neural Eng. 8:025005. doi: 10.1088/1741-2560/8/2/025005

Zander, T. O., Krol, L. R., Birbaumer, N. P., and Gramann, K. (2016). Neuroadaptive technology enables implicit cursor control based on medial prefrontal cortex activity. Proc. Natl. Acad. Sci. U.S.A. 113, 14898-14903. doi: 10.1073/pnas.1605155114

Conflict of Interest Statement: The authors declare that the research was conducted in the absence of any commercial or financial relationships that could be construed as a potential conflict of interest.

Copyright $(2017$ Nicolae, Acqualagna and Blankertz. This is an open-access article distributed under the terms of the Creative Commons Attribution License (CC BY). The use, distribution or reproduction in other forums is permitted, provided the original author(s) or licensor are credited and that the original publication in this journal is cited, in accordance with accepted academic practice. No use, distribution or reproduction is permitted which does not comply with these terms. 